\title{
Efficacy of Real-Time Continuous Glucose Monitoring to Improve Effects of a Prescriptive Lifestyle Intervention in Type 2 Diabetes: A Pilot Study
}

\author{
Penelope J. Taylor (D) - Campbell H. Thompson · Natalie D. Luscombe-Marsh • \\ Thomas P. Wycherley · Gary Wittert · Grant D. Brinkworth
}

Received: December 4, 2018 / Published online: January 31, 2019

(C) The Author(s) 2019

\begin{abstract}
Introduction: Optimising patient adherence to prescribed lifestyle interventions to achieve improved blood glucose control remains a challenge. Combined use of real-time continuous glucose monitoring systems (RT-CGM) may promote improved glycaemic control. This pilot study examines the effects of a prescriptive lifestyle modification programme when combined with RT-CGM on blood glucose control and cardiovascular disease risk markers.
\end{abstract}

Enhanced Digital Features To view enhanced digital features for this article go to https://doi.org/10.6084/ m9.figshare.7611449.

Electronic supplementary material The online version of this article (https://doi.org/10.1007/s13300019-0572-z) contains supplementary material, which is available to authorized users.

P. J. Taylor · N. D. Luscombe-Marsh

Commonwealth Scientific and Industrial Research Organisation, Health and Biosecurity, Adelaide, Australia

P. J. Taylor · C. H. Thompson · G. Wittert Discipline of Medicine, Adelaide Medical School, University of Adelaide, Adelaide, Australia

P. J. Taylor $(\varangle) \cdot$ N. D. Luscombe-Marsh · G. Wittert Nutrition and Metabolism, South Australian Health and Medical Research Institute (SAHRMI), Adelaide, Australia

e-mail: Pennie.Taylor@csiro.au
Methods: Twenty adults (10 men, 10 women) with obesity and type-2 diabetes (T2D) (age $60.55 \pm 8.38$ years, BMI $34.22 \pm 4.67 \mathrm{~kg} / \mathrm{m}^{2}$ ) were randomised to a prescriptive low-carbohydrate diet and lifestyle plan whilst continuously wearing either an RT-CGM or an 'offlineblinded' monitor (control) for 12 weeks. Outcomes were glycaemic control (HbA1c, fasting glucose, glycaemic variability [GV]), diabetes medication (MeS), weight, blood pressure and lipids assessed pre- and post-intervention.

Results: Both groups experienced reductions in body weight (RT-CGM $-7.4 \pm 4.5 \mathrm{~kg}$ vs. control $-5.5 \pm 4.0 \mathrm{~kg}), \mathrm{HbA} 1 \mathrm{c}(-0.67 \pm 0.82 \%$ vs. $-0.68 \pm 0.74 \%)$, fasting blood glucose $(-1.2 \pm$ $1.9 \mathrm{mmol} / \mathrm{L}$ vs. $-1.0 \pm 2.2 \mathrm{mmol} / \mathrm{L}), \quad$ LDL-C $(-0.07 \pm 0.34 \mathrm{mmol} / \mathrm{Lvs} .-0.26 \pm 0.42 \mathrm{mmol} / \mathrm{L})$ and triglycerides $(-0.32 \pm 0.46 \mathrm{mmol} / \mathrm{L}$ vs. $-0.36 \pm 0.53 \mathrm{mmol} / \mathrm{L})$; with no differential effect between groups $(P \geq 0.10)$. At week 12 , $\mathrm{GV}$ indices were consistently lower by at least

T. P. Wycherley

Alliance for Research in Exercise, Nutrition and Activity, School of Health Sciences, University of South Australia, Adelaide, Australia

G. D. Brinkworth

Commonwealth Scientific and Industrial Research Organisation, Health and Biosecurity, Sydney, Australia 
sixfold in RT-CGM compared to control (CONGA-1 $-0.27 \pm 0.36 \mathrm{mmol} / \mathrm{L}$ vs. $0.06 \pm$ $0.19 \mathrm{mmol} / \mathrm{L} ; \mathrm{CONGA}-2-0.36 \pm 0.54 \mathrm{mmol} / \mathrm{L}$ vs. $0.05 \pm 2.88 \mathrm{mmol} / \mathrm{L}$; CONGA-4 $-0.44 \pm$ $0.67 \mathrm{mmol} / \mathrm{L}$ vs. $\quad-\quad 0.02 \pm 0.42 \mathrm{mmol} / \mathrm{L}$; CONGA- $8-0.36 \pm 0.61$ vs. $0.02 \pm 0.52 \mathrm{mmol} /$ $\mathrm{L}$; MAGE $-0.69 \pm 1.14$ vs. $-0.09 \pm 0.08$ $\mathrm{mmol} / \mathrm{L}$, although there was insufficient power to achieve statistical significance $(P \geq 0.11)$. Overall, there was an approximately 40\% greater reduction in blood glucose-lowering medication $(\mathrm{MeS})$ in RT-CGM $(-0.30 \pm 0.59)$ compared to control $(0.02 \pm 0.23)$.

Conclusion: This study provides preliminary evidence that RT-CGM may be an effective strategy to optimise glucose control whilst following a low-carbohydrate lifestyle programme that targets improved glycaemic control, with minimal professional support.

Trial Registration: Australian New Zealand Clinical Trials Registry identifier, ANZTR: 372898.

Funding: Grant funding was received for the delivery of the clinical trial only, by the Diabetes Australia Research Trust (DART).

Keywords: Glycemic variability; Real-time continuous glucose monitoring; Type 2 diabetes

\section{INTRODUCTION}

Type 2 diabetes (T2D) poses an enormous socioeconomic burden [1]. It is associated with numerous vascular complications and a threeto sixfold increase in cardiovascular disease (CVD) risk. Optimising blood glucose levels remains a primary therapeutic target [2]. Despite modest effects, lifestyle modification incorporating diet and exercise remains the cornerstone of T2D management [2-6]. Lifestyle modification and particularly a low-carbohydrate diet can improve glycaemic control $[7,8]$ including reducing a patient's glycaemic variability (GV), i.e. the oscillations in blood glucose levels throughout the day [9]. GV has been identified as an independent risk factor for diabetes complications including CVD [1, 10-14]. Despite strong efficacy of lifestyle modification programmes, their effectiveness is often underpinned by intensive techniques requiring close monitoring and health professionals' support to achieve adherence and desired health outcomes [15]. These practice models are therefore resource-intensive and cost-prohibitive, limiting their widespread availability.

Self-regulation that enables a patient to exert confidence and control over their diet and exercise behaviours is a key component to effective lifestyle intervention adherence $[16,17]$. One approach to enhance patient self-regulation is to provide them with immediate feedback based on the results of their behaviours [18]. Self-monitoring of health markers and behaviours beyond the clinical setting has been used as an effective tool to monitor treatment response and improve adherence for a variety of health outcomes including body weight, blood pressure and physical activity [18]. This suggests that selfmonitoring of blood glucose (SMBG) in T2D could serve as an immediate feedback function to provide patients with evidence of the biological effect of lifestyle choices on blood glucose levels that may improve adherence to lifestyle prescription and improve glycaemic outcomes [19-21]. Real-time continuous glucose monitoring (RT-CGM), which provides an ongoing display of glucose levels over an extended period (i.e. days/weeks), allows an individual to selfmonitor how their blood glucose level responds to various lifestyle factors including diet and exercise, with direct and continuous dynamic feedback. It is therefore possible that RT-CGM might motivate and guide patients to adapt their lifestyle patterns in an appropriate manner to reduce GV and improve glycaemic control. Previous studies have shown that RT-CGM can promote an approximately $0.5 \%$ (absolute) greater reduction in HbA1c compared to conventional SMBG using the finger-prick method in T2D [20, 22-25]. However, these studies did not comprehensively assess the effects of RTCGM on GV or combine this therapeutic strategy within a comprehensive and structured diet and lifestyle programme that targets minimisation of wide glucose fluctuations. Consequently, previous studies have required patients to make changes to diet and exercise in response to SMBG in the absence of advice on those changes that will be most effective to optimise glycaemic control. The purpose of this feasibility study was 
to examine the effects of RT-CGM compared to blinded CGM on blood glucose control as assessed by HbA1c, GV and CVD risk markers when undertaking a prescriptive lifestyle modification programme that has minimal healthcare practitioner involvement.

\section{METHODS}

\section{Study Participants}

Twenty adults who were overweight or obese (BMI $26-45 \mathrm{~kg} / \mathrm{m}^{2}$, age $20-75$ years) with T2D [HbA1c 5.9-6.9\% (41.0-51.9 $\mathrm{mmol} / \mathrm{mol})]$ were recruited via public advertisement to participate in a randomised controlled study, conducted between June and September 2017 at the Commonwealth Scientific and Industrial Research Organisation (CSIRO), Health and Nutrition Research Unit (Adelaide Australia), Table 1, Fig. 1 (participant flow). Exclusion criteria were type 1 diabetes; proteinuria (urinary albumin-to-creatinine ratio $\geq 30 \mathrm{mg} / \mathrm{mmol}$ ), abnormal liver function [alanine aminotransferase (ALT), aspartate aminotransferase (AST) or gamma-glutamyl transferase (GGT) $\geq 2.5$ times the normal upper limit], impaired renal function (eGFR $<60 \mathrm{ml} / \mathrm{min}$ ), any abnormal or significant clinical history including current malignancy, liver, respiratory, gastrointestinal, cardiovascular disease or pregnancy/lactation, eating disorder or clinical depression; any significant endocrinopathy (other than stable treated thyroid disease); have taken/or taking glucocorticoids (oral/inhaled or topical) within last 3 months, psychotropics other than a stable dose of a selective serotonin reuptake inhibitor; illicit drugs, medications which affect gastrointestinal motility or hunger/appetite (e.g. metoclopramide, domperidone and cisapride, anticholinergic drugs (e.g. atropine), erythromycin) or past history of gastrointestinal surgery which may affect study outcomes. All participants provided written informed consent prior to participation. The study was registered with the Australian New Zealand Clinical Trials Registry (ANZTR 372898) and approved by the Human Research Ethics Committees of the CSIRO and the University of Adelaide.

\section{Study Design and Intervention}

This was a feasibility pilot study, i.e. a smallscale investigation that was conducted and published to inform researchers of important parameters and sample size requirements required for an adequately powered randomised control trial. In a parallel design, participants were matched for age and gender and randomised using a computer-generated randomisation procedure (www.randomisation.com) to undertake a 12-week lifestyle (diet and exercise) intervention with either (1) real-time continuous glucose monitoring (RT-CGM) with access to visual display or (2) continuous glucose monitoring (blinded CGM; control), with no access to visual display. Randomisation (sequence generation) was performed by the clinical trials manager, who was unblinded for the purpose of providing device and technology support, including technical troubleshooting for device connectivity and the administration of sensor kits to the participants. All other research associates responsible for data collection, processing and analysis were blinded until data analysis was complete. Participants received a once-off honorarium (\$200 AUD) for trial participation.

At week 0 (after the completion of baseline assessments), participants in both groups received a prescriptive low-carbohydrate, highprotein and unsaturated fat diet (LC diet) and exercise plan, incorporating moderate intensity aerobic/resistance exercises in the form of a commercial publication; at this point randomisation was revealed to participants and to primary staff responsible for administration of glucose sensors and downloads [26]. The dietary prescription had a planned macronutrient profile of $14 \%$ of total energy as carbohydrate, $28 \%$ protein and $58 \%$ total fat (35\% monounsaturated fat), individualised for energy level based on achieving a 30\% energy restriction. This dietary profile and lifestyle programme have been previously demonstrated to promote weight loss and enhance glycaemic control and cardiovascular disease risk markers $[7,8]$. At week 3 , participants were provided with education on food exchanges and provided lists of alternative foods, based on similar nutrient and 


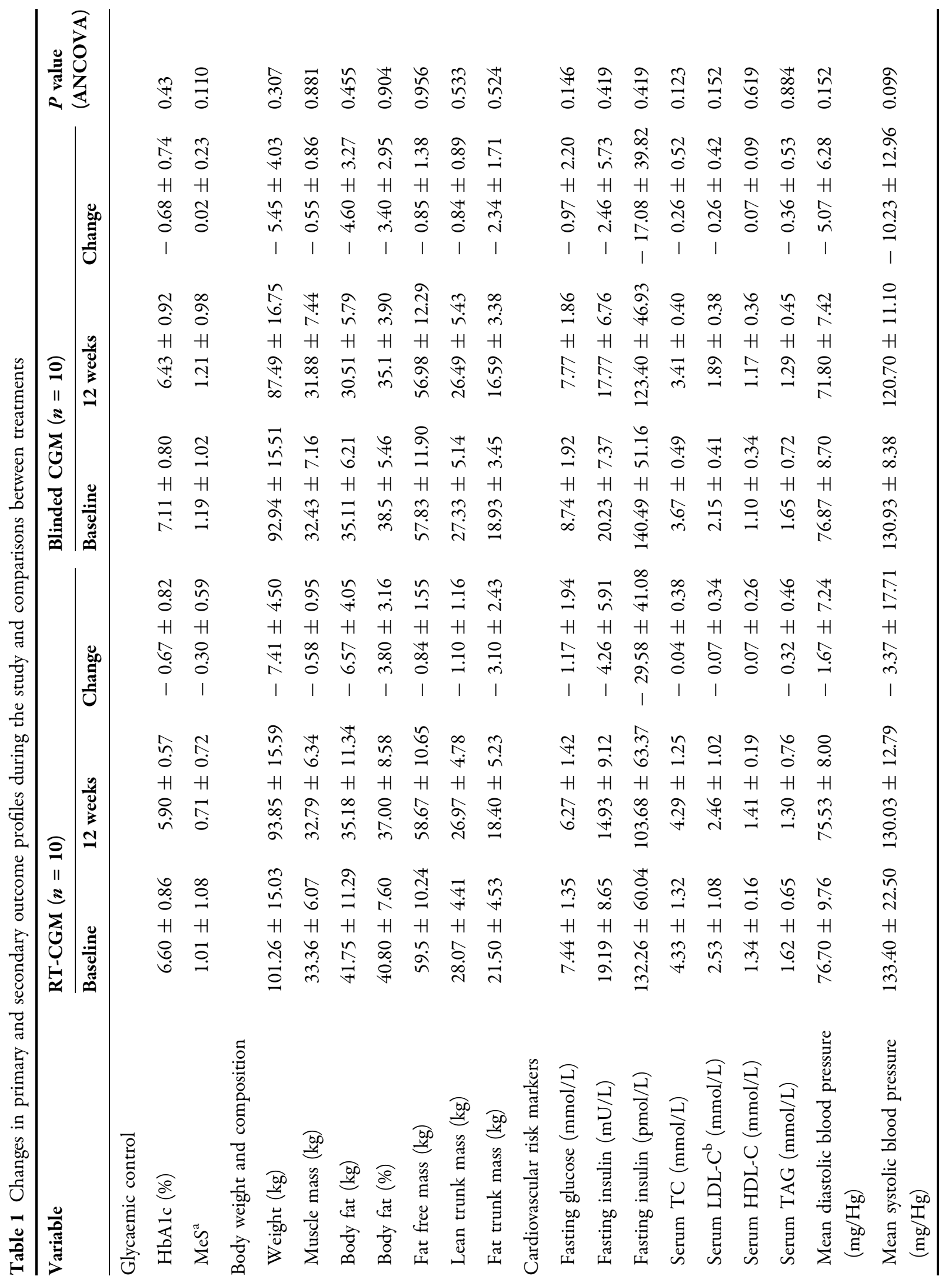




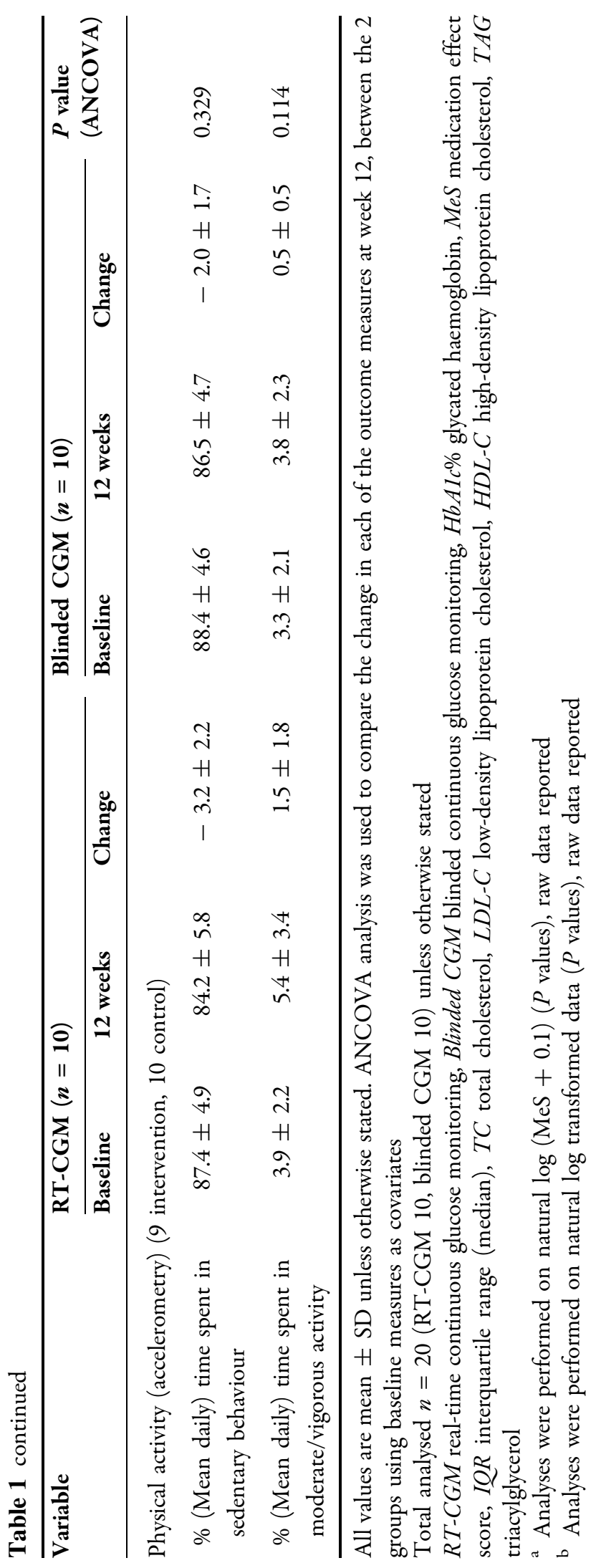




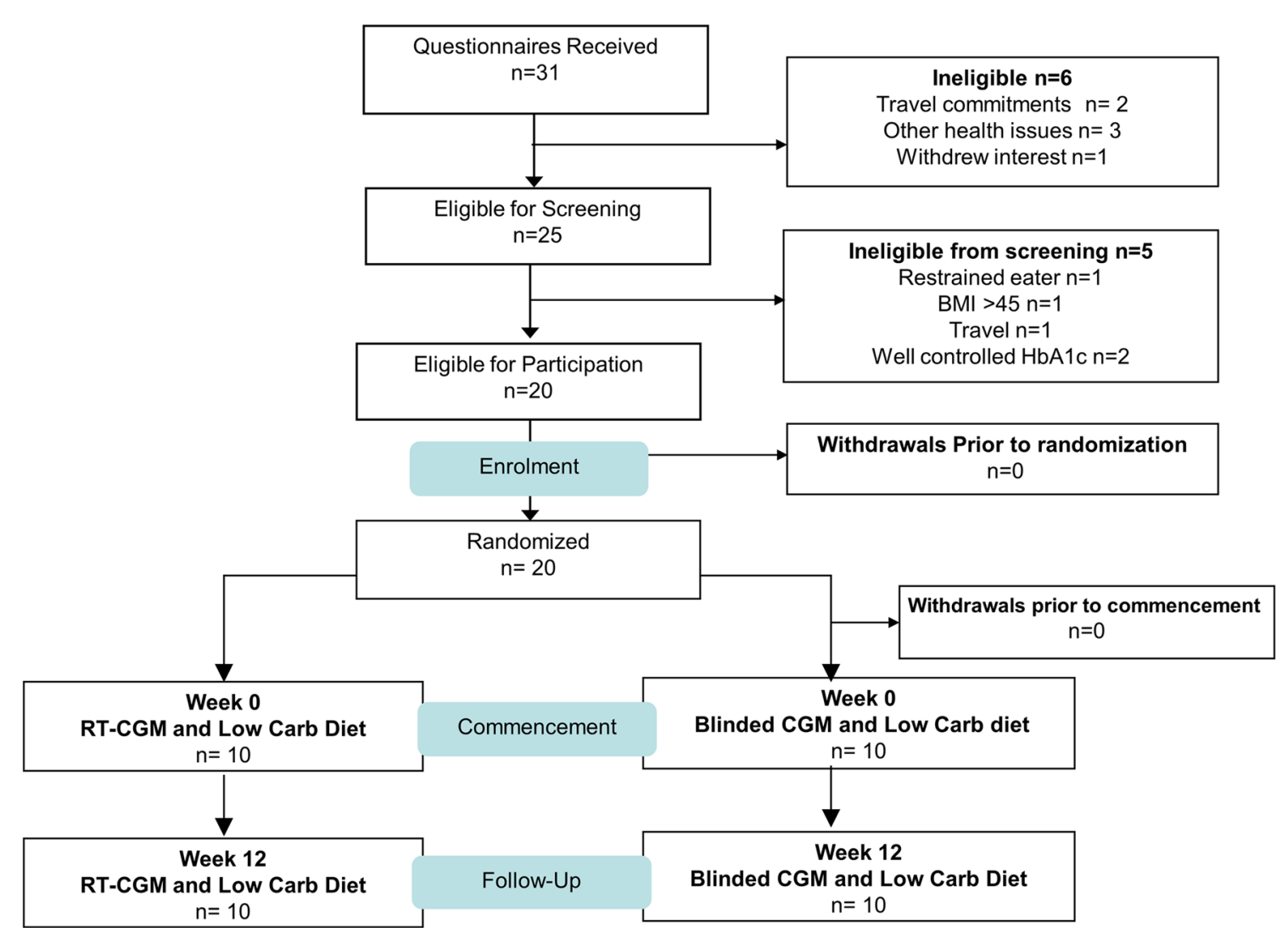

Fig. 1 Participant flow

energy density of foods within the dietary benchmarks, to assist participants with making suitable food substitutions to improve their dietary flexibility. Use of the materials was selfregulated. Participants received no further formal lifestyle counselling regarding the diet and exercise plan.

Both groups were instructed to perform usual SMBG readings before and after each meal and at bedtime, as per standard practice. Blood glucose monitoring logs were kept and participants were provided with glucometers and testing strips to promote compliance. To facilitate compliance with CGM device wear, at commencement of the study, participants met with a research nurse and dietitian who provided training with practice demonstrations on how insert, initiate, calibrate and change the glucose sensor every 10 days over the 12 -week period. Contact details for the research nurse were provided to participants for remote device support and to answer any queries on insertion, initiation and changing of the sensor during the intervention. Participants visited the clinic every 3 weeks for the research nurse to download sensor glucose data, check on glucose sensor insertion and initiation technique, review morning fasting glucose logs, and to replenish devices supplies.

\section{Outcome Measures}

Outcomes were assessed at baseline (week 0) and end of intervention (week 12). The primary outcomes were HbA1c (Clinpath, Adelaide, Australia), diurnal GV [7, 27] and diabetes-related medication changes assessed by the antiglycaemic medication effect score (MeS) $[7,28,29]$. The anti-glycaemic medication effect score is an overall assessment of the total use of anti-glycaemic agents, based on type and dose of agents, with the high scores corresponding to higher anti-glycaemic medication usage. This calculation takes into consideration each prescribed drug by daily patient dose and is 
expressed as a percentage of maximum recommended daily dose of that drug. This percentage is then multiplied by an adjustment factor, e.g. 1.5 for sulfonylureas and biguanides and 2.5 for insulin. For individuals prescribed more than one anti-glycaemic agent, each agent's daily dose is multiplied by the respective adjustment factor and outcomes aggregated to generate the final MeS [28-30]. For example, at baseline one participant was prescribed $1000 \mathrm{mg}$ and $500 \mathrm{mg}$ of an oral hypoglycaemic agent, metformin, in the morning and night, respectively, for a total daily dose of $1500 \mathrm{mg}$. Maximum daily dose for this medication is $3000 \mathrm{mg}$, and therefore the participant's dose for this medication was $50 \%$. This value is then multiplied by the relevant drug's adjustment factor, 1.5 in this example, providing an overall baseline MeS of 0.75 . At trial completion (week 12), this participant had experienced a dose reduction in metformin to $500 \mathrm{mg}$ twice daily resulting in a total daily dose of $1000 \mathrm{mg}$ (33\% of the drugs maximum daily dose) and a MeS of 0.50. To determine the change in the MeS (and medication intensity), the post-study MeS (0.50) is subtracted from baseline MeS (0.75) to provide an overall absolute reduction in $\mathrm{MeS}$ and medication intensity of 0.25 .

Secondary outcomes included weight and body composition assessed by bioelectrical impedance (InBody 230, InBody Co. Ltd. South Korea), fasting blood lipids (total cholesterol, triglycerides and HDL-cholesterol), glucose and insulin (Clinpath, Adelaide, Australia) with LDL-cholesterol calculated using the modified Friedewald equation [31] and blood pressure was measured using an automated sphygmomanometer (SureSigns Vs3, Phillips Medical Systems, MA, USA); and physical activity levels assessed using seven consecutive days of ambulatory accelerometer monitoring (GT3X+; ActiGraph, Pensacola, FLA).

\section{Continuous Glucose Monitoring, Glycaemic Variability and Medication Effect Score}

Participants wore the Medtronic ${ }^{\mathrm{TM}}$ Guardian Connect $^{\circledR}$ device with the Harmony ${ }^{\circledR}$ glucose sensor (Medtronic, Los Angeles, CA, USA) continuously for 13 weeks. All participants were blinded CGM for 1 week prior to commencement of the lifestyle intervention for baseline data collection, then randomisation revealed at baseline (week 0) for 12 weeks whilst following the lifestyle intervention. Devices were calibrated according to the manufacturer's recommendations with a sensor change every 10 days. Participants in the RT-CGM group received an iPod device (Apple iPod Nano, portable medial player, Cupertino, CA) which was Bluetooth connected to the CGM to provide real-time blood glucose level displays throughout the 12-week intervention period. All participants were asked to perform SMBG first thing in the morning before breakfast (fasting) and before each meal. Participants were provided with AccuChek $^{\mathrm{TM}}$ glucometers (Roche Diagnostics, Sydney Australia) and testing strips, to replicate standard care.

Diurnal glucose profiles, derived from interstitial fluid readings performed every $5 \mathrm{~min}$ over back-to-back 10-day cycles for 91 days (i.e. 13 weeks), were collected using the CGM device. CGM data for the 7 days prior to intervention commencement and the final 7 days of the intervention were used as the pre- and poststudy outcomes respectively to compute GV measures including mean, maximum and minimum glucose levels; standard deviation of glucose $\left(\mathrm{SD}_{\text {intraday }}\right)$; mean amplitude of glycaemic excursions (MAGE); continuous overall net glycaemic action (CONGA-1, 2, 4 and 8); overall percentage of total time spent in euglycaemia (3.9-10 $\mathrm{mmol} / \mathrm{l})$, hyperglycaemia $(>10.0 \mathrm{mmol} / \mathrm{L})$ and hypoglycaemia $(<3.9 \mathrm{mmol} / \mathrm{L})$ were calculated as per glycaemic control targets described by the American Diabetes Association (ADA) [2].

At baseline and throughout the study, blood sugar-lowering medication type, dosage and changes were monitored and documented. The anti-glycaemic MeS was calculated to assess overall utilisation of blood sugar-lowering medication, with higher MeS corresponding to higher usage of blood sugar-lowering medication $[28,29]$. 


\section{Statistical Analysis}

Statistical analysis was conducted using SPSS Statistics 25 (IBM Corp, 2017). Analysis of covariance (ANCOVA) was used to test betweengroup differences at 12 weeks, using baseline measures as covariates [32]. The model residuals were examined for normality and constant variance. Where these assumptions were not met, transformations of the variables were considered. Log (natural) transformation improved the distributional assumptions for serum LDL-C, GV indices and MeS, and $P$ values from the transformed analyses are reported. Statistical significance was determined at $P<0.05$. Data are presented as mean \pm SD.

\section{RESULTS}

Twenty participants commenced and completed the study (Fig. 1). Baseline characteristics were similar between groups (mean $\pm \mathrm{SD}$; RTCGM vs blinded CGM): age $60.2 \pm 8.8$ years vs. $60.9 \pm 8.4$ years, diabetes duration $10.5 \pm$ 7.3 years vs. $11.0 \pm 4.1$ years, HbA1c $6.6 \pm 0.9 \%$ vs. $7.1 \pm 0.8 \% \quad(49 \pm 2 \mathrm{mmol} / \mathrm{mol} \quad$ vs. $54 \pm$ $2 \mathrm{mmol} / \mathrm{mol}$ ) with an even gender distribution ( 5 men/5 women in both groups) (see Table S1 in the supplementary material).

Changes from baseline (mean $\pm \mathrm{SD}$ ) are reported in Table 1 (see supplementary material and Tables 1, 2 and 3 for changes in primary and secondary outcome profiles during the study and comparisons between treatments). After week 12, body weight, body fat, HbA1c, fasting glucose, insulin, blood lipids (total cholesterol, LDL-C, triglycerides and HDL-C) and blood pressure were not statistically different between groups $(P \geq 0.10)$.

Participants wore the CGM device for 84 consecutive days, with wear-time adherence at $100 \%$ for all but one participant who removed the device for aquatic activities for 3 days only. GV was assessed in 15 participants (RT-CGM, $n=9$; blinded CGM, $n=6$ ). Sufficient data, at week 12 , were not collected for $n=5$ participants secondary to reduced connectivity between glucose sensor and glucose recorder. No statistically significant differences between the groups at week 12 occurred for markers of GV including MAGE, CONGA-1, CONGA-8 and SD or anti-glycaemic MeS $(P \geq 0.11)$. Despite the lack of statistical significance, at week $12, \mathrm{GV}$ indices were consistently $20-25 \%$ lower in the RT-CGM group compared with the blinded CGM group. Post hoc power analysis determined that a minimum of 35 participants per group would have been needed to achieve statistical significance. This was based on determining, for a parallel group superiority trial, the number of participants required in two randomised groups to have an $80 \%$ chance of detecting as significant at the $5 \%$ level the minimum difference between the groups means observed for the GV outcomes in the present study.

The level of diabetes medication, as reflected by the anti-glycaemic MeS, was $40 \%$ lower in the RT-CGM compared to the blinded CGM group at week 12. Thirteen participants had no change in anti-glycaemic MeS (RT-CGM, $n=7$; blinded CGM, $n=6$ ). In the RT-CGM group, three participants experienced a reduction in MeS with no participants experiencing an increase. In the blinded CGM group, two participants experienced a decrease in $\mathrm{MeS}$ and two participants experienced an increase.

Percentage time spent in sedentary behaviour and percentage time spent in moderate/ vigorous activity were similar in both groups $(P \geq 0.11)$ at 12 weeks.

\section{DISCUSSION}

This pilot study examined the effects of RTCGM with visual feedback compared to blinded CGM, with no visual feedback in individuals with T2D when undertaking a low-intensity, prescriptive low-carbohydrate diet and lifestyle plan. Despite the apparent lack of statistical power, this study provides preliminary evidence that access to RT-CGM feedback is an effective approach to reinforce the effects of lifestyle modification strategies to improve diabetes control by reducing GV and diabetes medication requirements. The high level of device wear-time adherence also suggests a good tolerability to the device usage. 


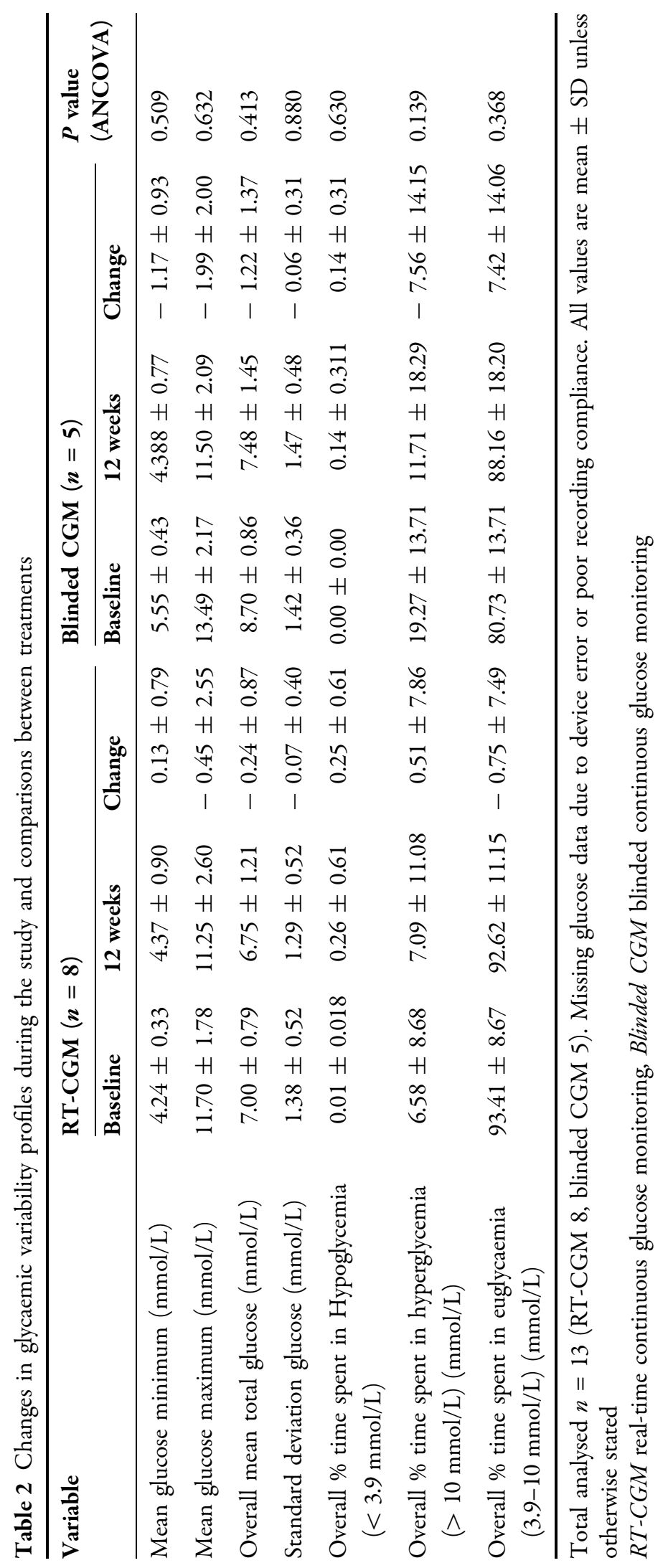


Table 3 Changes in glycaemic variability profiles for MAGE, CONGA-1, 2, 4 and 8 during the study and comparisons between treatments

\begin{tabular}{|c|c|c|c|c|c|c|c|}
\hline \multirow[t]{2}{*}{ Variable } & \multicolumn{3}{|c|}{ RT-CGM $(n=9)$} & \multicolumn{3}{|c|}{ Blinded CGM $(n=6)$} & \multirow{2}{*}{$\begin{array}{l}P \text { value } \\
\text { (ANCOVA) }\end{array}$} \\
\hline & Baseline & 12 weeks & Change & Baseline & 12 weeks & Change & \\
\hline $\begin{array}{l}\text { CONGA-1 } \\
(\mathrm{mmol} / \mathrm{L})\end{array}$ & $1.30 \pm 0.36$ & $1.03 \pm 0.36$ & $-0.27 \pm 0.36$ & $1.24 \pm 0.41$ & $1.30 \pm 0.48$ & $0.06 \pm 0.19$ & 0.074 \\
\hline $\begin{array}{l}\text { CONGA-2 } \\
(\mathrm{mmol} / \mathrm{L})\end{array}$ & $1.72 \pm 0.51$ & $1.36 \pm 0.55$ & $-0.36 \pm 0.54$ & $1.79 \pm 0.633$ & $1.84 \pm 0.77$ & $0.05 \pm 2.88$ & 0.110 \\
\hline $\begin{array}{l}\text { CONGA-4 } \\
(\mathrm{mmol} / \mathrm{L})\end{array}$ & $2.02 \pm 0.68$ & $1.58 \pm 0.75$ & $-0.44 \pm 0.67$ & $2.18 \pm 0.74$ & $2.16 \pm 0.93$ & $-0.02 \pm 0.42$ & 0.186 \\
\hline $\begin{array}{l}\text { CONGA-8 } \\
(\mathrm{mmol} / \mathrm{L})\end{array}$ & $2.09 \pm 0.68$ & $1.73 \pm 0.83$ & $-0.36 \pm 0.61$ & $2.25 \pm 0.73$ & $2.22 \pm 0.95$ & $-0.02 \pm 0.52$ & 0.298 \\
\hline $\begin{array}{l}\text { MAGE } \\
(\mathrm{mmol} / \mathrm{L})\end{array}$ & $3.69 \pm 1.08$ & $3.01 \pm 1.44$ & $-0.69 \pm 1.14$ & $4.06 \pm 1.23$ & $4.05 \pm 1.61$ & $-0.09 \pm 0.80$ & 0.250 \\
\hline
\end{tabular}

Total analysed $n=15$ (RT-CGM 9, blinded CGM 6). Missing glucose data due to device recording or sensor insertion error. All values are mean $\pm S D$ unless otherwise stated

RT-CGM real-time continuous glucose monitoring, Blinded CGM blinded continuous glucose monitoring, CONGA-1 continuous overall net glycaemic action of observations $1 \mathrm{~h}$ apart, CONGA-2 continuous overall net glycaemic action of observations 2-h apart, CONGA-4 continuous overall net glycaemic action of observations $4 \mathrm{~h}$ apart, CONGA-8 continuous overall net glycaemic action of observations $8 \mathrm{~h}$ apart, $M A G E$ mean amplitude of glycaemic excursions

Overall, both groups achieved approximately $6 \%$ weight loss which is considered clinically relevant and comparable to most structured weight loss programmes that typically involve intensive counselling compared to this study that provided limited professional support $[4,33]$. The lifestyle intervention in the present study used a low-carbohydrate-based prescriptive meal plan combined with a prescriptive aerobic/resistance-based exercise programme presented in a book format. This demonstrates that provision of a highly structured, prescriptive lifestyle plan can be an effective strategy to promote weight loss without the necessity of intensive professional support.

Together with weight loss, both groups experienced an average $0.7 \% \mathrm{HbA} 1 \mathrm{c}$ reduction. This is consistent with other weight loss studies in T2D of similar study duration [20, 23, 34]. A recent meta-analysis demonstrated an estimated mean $\mathrm{HbA} 1 \mathrm{c}$ reduction of $0.1 \%$ for each $1 \mathrm{~kg}$ of reduced body weight in this population [35]. For every $1 \%$ reduction in HbA1c there is an expected $37 \%$ reduced risk for microvascular complications and $21 \%$ reduction in the risk of premature death related to T2D [33]. This highlights the clinical significance of the changes observed in the present study.

Beyond HbA1c, which reflects average blood glucose levels over approximately 3 months, GV relates to fluctuations in blood glucose levels across the day or between days [36, 37]. GV has been identified as an independent risk factor of T2D-related micro- and macrovascular complications. Intermittent high blood glucose spikes, as opposed to constant exposure to high blood glucose, has been shown to promote damaging effects $[10,12,38]$. Daily glucose fluctuations are incompletely expressed by HbA1c alone, particularly in patients considered to have good metabolic control or with prediabetes $[9,13,36,39,40]$. To date, few studies have examined the effects of RT-CGM on GV. Yoo et al. demonstrated a $22 \%$ reduction in $\mathrm{GV}$ as measured by MAGE following the use of RTCGM (worn for 3 days a month for 3 months) in 65 individuals with poorly controlled T2D (HbA1c $>8 \%)$ [20]. In this study no control 
group comparison was made. Similarly, in the present study, GV was approximately $20-25 \%$ lower in the RT-CGM compared to the blindedCGM control after the intervention. However, as a result of the small sample size and lack of statistical significance, these data need to be interpreted with caution.

Nonetheless, these data suggest that RTCGM may assist individuals to minimise blood glucose fluctuations and, given the emerging clinical importance of GV for promoting diabetes-related complications, larger studies are required to confirm these effects. A larger study could also investigate differences in the trajectories in the GV response over the intervention period. Additionally, since the individuals studied were in reasonable glycaemic control at baseline (mean HbA1c $<7 \%$ and a high proportion of time spent in the euglycaemic range) this may have moderated the degree of improvement observed. Hence, further investigation of poorly controlled individuals that may experience more amplified improvements warrants further investigation. Larger future trials could use an intention to treat design and appropriate analysis to mitigate completer bias.

Despite commencing with a lower MeS, the RT-CGM group experienced a greater reduction in MeS compared to the blinded CGM group such that MeS was $40 \%$ lower in RT-CGM at week 12 . However, it is important to note that these differential group changes were driven by medication changes in only a few individuals. Hence, these observations need to be treated with great caution. Nonetheless, despite the clinical benefits of intensive hypoglycaemic medication prescription for reducing macrovascular and microvascular disease risk through HbA1c reduction [2], a recent report suggests that HbA1c reduction alone may not reduce macrovascular endpoints [41, 42] and the side effects of pharmacotherapy are well known $[43,44]$. Thus it is necessary to examine the potential of RT-CGM to alter medication prescription in larger populations, particularly in highly medicated individuals.

It is well known that lifestyle therapies are effective in T2D management but adherence is difficult and that negatively impacts effectiveness $[15,40,45]$. The preliminary data from the current study suggest that instant access to feedback regarding their daily glucose levels may improve patients' adherence and sustainability of lifestyle changes to optimise glucose control $[40,46]$. This is supported by a recent systematic review providing robust evidence suggesting that engagement in self-management education had a most favourable effect on glycaemic control [45]. However, debate exists on the duration and frequency of CGM data exposure that are required to modify behaviour and achieve clinically relevant improvements. Further studies should also examine the dose-response effect of exposure to RT-CGM data for the improvement of diabetes control.

The present study had several limitations. Firstly, this was a pilot study and, despite the promising magnitude of the differences observed between groups, results should be treated with caution and these promising results warrant further investigation with larger trials. The study also examined individuals with relatively good glycaemic control and future studies examining larger populations with wider levels of glucose control would allow a better understanding of the applicability of RTCGM for T2D management. Additionally, evaluation of various drug types on markers of GV or MeS was not performed. Previous studies have demonstrated that an increase in oral hypoglycaemic medications may sometimes result in a drop in MAGE [40]. To better understand the clinical implications of RT-CGM on GV, future trials should closely monitor and consider medication types, doses and changes in dose over the time course of the intervention. Some difficulties with device connectivity between sensor and recorder in the blinded CGM group were also experienced and future studies should specifically examine whether this is related to the blinded use of the CGM device. Finally, our retention rate was high, and it cannot be ruled out that provision of an honorarium, which is not reflective of current clinical practice, could have potentially influenced participant compliance to the intervention. 


\section{CONCLUSION}

This study provides preliminary evidence that RT-CGM may enhance the benefits of a prescriptive low-carbohydrate diet and exercise plan delivered with minimal professional support, improving glycaemic control by reducing daily GV. These pilot findings provide a rationale for more comprehensive, larger-scale randomised controlled trials to be conducted. Trials should also consider the duration and frequency of sensor-wear time, medication types and changes in medication over longerterm interventions in order to better assess this therapeutic technology.

\section{ACKNOWLEDGEMENTS}

The authors would like to thank all study participants and their families for their participation in the study.

Funding. Grant funding was received for the delivery of the clinical trial only, by the Diabetes Australia Research Trust (DART). No funding was received for preparation or publication of this article, these were funded by the authors.

Additional Assistance. The authors wish to thank Julia Weaver, Anne McGuffin, Vanessa Courage, Dr Eva Pederson and Theresa McKinnon for their support during the study and to Julie Syrette for supporting the preparation of the glucose data for this analysis. A heartfelt thank you is extended to the Taylor family, Paul, Mitchell, Skye and Harry for their patience during this year, and for the support you have provided.

Authorship. All authors meet the International Committee of Medical Journal Editors authorship guidelines (ICMJE) for this article, all had access to the data obtained in this study and take responsibility for the integrity of the data and accuracy of the data analysis.

Disclosures. All authors, Pennie Taylor, Campbell Thompson, Grant Brinkworth,
Natalie Luscombe-Marsh, Thomas Wycherley and Gary Wittert state that they have no conflict of interest to declare.

Compliance with Ethics Guidelines. Ethics approval for the conduct of this clinical trial was provided by The Adelaide University Human Research Ethics Committee and the study has conformed to the Helsinki Declaration 1964, as reviewed by 2013, concerning human rights. Springers policy concerning informed consent has been followed. All procedures performed in this study were in accordance with the ethics standards of the Adelaide University Human Ethics and Research Committee and with the 1964 Helsinki Declaration and its later amendments. Informed consent was obtained from all individual participants included in the study.

Data Availability. The datasets analyzed during the current study are available from the corresponding author on reasonable request.

Open Access. This article is distributed under the terms of the Creative Commons Attribution-NonCommercial 4.0 International License (http://creativecommons.org/licenses/ by-nc/4.0/), which permits any noncommercial use, distribution, and reproduction in any medium, provided you give appropriate credit to the original author(s) and the source, provide a link to the Creative Commons license, and indicate if changes were made.

\section{REFERENCES}

1. Maurizi AR, Pozzilli P. Do we need continuous glucose monitoring in type 2 diabetes? Diabetes Metab Res Rev. 2013. https://doi.org/10.1002/dmrr. 2450 .

2. American Diaebtes Associated (ADA): Standards of Medical Care in Diabetes-2017. Diab Care. 2017;40 (Suppl 1):S1-S5.

3. Dunkley AJ, Charles K, Gray LK, et al. Effectiveness of interventions for reducing diabetes and cardiovascular disease risk in people with metabolic syndrome: systematic review and mixed treatment 
comparison meta-analysis. Diabetes Obes Metab. 2012;14:616-25.

4. Terranova CO, Brakenridge CL, Lawler SP, et al. Effectiveness of lifestyle-based weight loss interventions for adults with type 2 diabetes: a systematic review and meta-analysis. Diabetes Obes Metab. 2015;17:371-8.

5. Franz MJ, Boucher JL, Rutten-Ramon S, et al. Lifestyle weight-loss intervention outcomes in overweight and obese adults with type 2 diabetes: a systematic review and meta-analysis of randomized clinical trials. J Acad Nutr Diet. 2015;115:1447-63.

6. Forouhi NG, Misra A, Mohan V, et al. Dietary and nutritional approaches for prevention and management of type 2 diabetes. BMJ. 2018;361:2234-43.

7. Tay J, Luscombe-Marsh ND, Thompson $\mathrm{CH}$, et al. Comparison of low- and high-carbohydrate diets for type 2 diabetes management: a randomized trial. Am J Clin Nutr. 2015;102:780-90.

8. Tay J, Luscombe-Marsh ND, Thompson $\mathrm{CH}$, et al. A very low-carbohydrate, low-saturated fat diet for type 2 diabetes management: a randomized trial. Diabetes Care. 2014;37:2909-18.

9. Suh S, Kim JH. Glycemic variability: how do we measure it and why is it important? Diabetes Metab J. 2015;39:273-82.

10. Cardoso CRL, Leite NC, Moram CBM, et al. Longterm visit-to-visit glycemic variability as predictor of micro- and macrovascular complications in patients with type 2 diabetes: The Rio de Janeiro Type 2 Diabetes Cohort Study. Cardiovasc Diabetol. 2018;17-33.

11. Skrha J, Soupal J, Skrha JJR, et al. Glucose variability, HbA1c and microvascular complications. Rev Endocr Metab Disord. 2016;17:103-10.

12. Monnier L, Mas E, Ginet C, et al. Activation of oxidative stress by acute glucose fluctuations compared with sustained chronic hyperglycemia in patients with type 2 diabetes. JAMA. 2006;295:1681-7.

13. Gorst C, Kwok CS, Aslam S, et al. Long term GV and risk of adverse outcomes. Diabetes Care. 2015;38:2354-69. https://doi.org/10.2337/dc151188 .

14. Hirsch IB. Glycemic variability and diabetes complications: does it matter? Of course it does! Diabetes Care. 2015;38:1610-4.

15. Wens J, Vermeire E, Royen PV, et al. GPs' perspectives of type 2 diabetes patients' adherence to treatment: a qualitative analysis of barriers and solutions. BMC Fam Pract. 2005;6:20-30.

16. Samdal GB, Eide GE, Barth T, et al. Effective behaviour change techniques for physical activity and healthy eating in overweight and obese adults; systematic review and meta-regression analyses. Int J Behav Nutr Phys Act. 2017;4:42-6.

17. Guerci B, Drouin P, Grange V, et al. Self-monitoring of blood glucose significantly improves metabolic control in patients with type 2 diabetes mellitus: the Auto-Surveillance Intervention Active (ASIA) study. Diab Metab. 2003;29:587-94.

18. Michie S, Abrahan C, Whittington C, et al. Effective techniques in healthy eating and physical activity interventions: a meta-regression. Health Psychol. 2009;28:690-701.

19. McAndrew L, Schneider SH, Burns E, et al. Does patient blood glucose monitoring improve diabetes control? A systematic review of the literature. Diabetes Educ. 2007;33:991-1011.

20. Yoo HJ, An HG, Park SY, et al. Use of a real time continuous glucose monitoring system as a motivational device for poorly controlled type 2 diabetes. Diabetes Res Clin Pract. 2008;82:73-9.

21. Bailey KJ, Little PJ, Jung ME. Self-monitoring using continuous glucose monitors with real-time feedback improves exercise adherence in individuals with impaired blood glucose: a pilot study. Diabetes Technol Ther. 2016;18:185-93.

22. Yoo HJ, Kim HS, Yang SJ, et al. Use of real time continuous glucose monitoring system as a motivational device for type 2 diabetes. Diabetes. 2008;57:55.

23. Ehrhardt NM, Chellappa M, Walker MS, et al. The effect of real-time continuous glucose monitoring on glycemic control in patients with type 2 diabetes mellitus. J Diabetes Sci Technol. 2011;5:668-75.

24. Vigersky RA, Fonda SJ, Chellappa M, et al. Shortand long-term effects of real-time continuous glucose monitoring in patients with type 2 diabetes. Diabetes Care. 2012;35:32-8.

25. Poolsup N, Suksomboon N, Kyaw AM. Systematic review and meta-analysis of the effectiveness of continuous glucose monitoring (CGM) on glucose control in diabetes. Diabetol Metab Syndr. 2013;5:39-45.

26. Brinkworth GD, Taylor PJ. The CSIRO low carb diet. Sydney: Pan Macmillian; 2017.

27. Tay J, Thompson $\mathrm{CH}$, Brinkworth GD. Glycemic variability: assessing glycemia differently and the 
implications for dietary management of diabetes. Annu Rev Nutr. 2015;35:389-424.

28. Mayer SB, Jeffreys AS, Olsen MK, et al. Two diets with different haemoglobin A1c and antiglycaemic medication effects despite similar weight loss in type 2 diabetes. Diabetes Obes Metab. 2014;16:90-3.

29. Nathan DM, Buse JB, Davidson MB, et al. Medical management of hyperglycemia in type 2 diabetes: a consensus algorithm for the initiation and adjustment of therapy: a consensus statement of the American Diabetes Association and the European Association for the Study of Diabetes. Diabetes Care. 2009;32:93-203.

30. Taylor PJ, Lange K, Thompson $\mathrm{CH}$, et al. Association of glycemic variability and the anti-glycemic medication effect score in adults with type 2 diabetes. Diabetes Management. 2018;8:117-27.

31. Chen Y, Zhang X, Pan B, et al. A modified formula for calculating low-density lipoprotein cholesterol values. Lipids Health Dis. 2010;9:52.

32. Bland MJ, Altman DG. Best (but oft forgotten) practices: testing for treatment effects in randomized trials by separate analyses of changes from baseline in each group is a misleading approach. Am J Clin Nutr. 2015;102:991-4.

33. Stratton IM, Neil Adler AA, Beil AW, et al. Association of glycaemia with macrovascular and microvasular complications of type 2 diabetes (UKPD 35): prospective observational study. BMJ. 2000;321:405-12.

34. Odgers-Jewell K, Ball LE, Kelly JT, et al. Effectiveness of group-based self-management education for individuals with type 2 diabetes: a systematic review with meta-analyses and meta-regression. Diabet Med. 2017;34:1027-39.

35. Gummesson A, Nyman E, Knutsson M, et al. Effect of weight reduction on glycated haemoglobin in weight loss trials in patients with type 2 diabetes. Diabetes Obes Metab. 2017;19:1295-305.

36. Frontoni S, Di Bartolo P, Avogaro A, et al. Glucose variability: an emerging target for the treatment of diabetes mellitus. Diabetes Res Clin Pract. 2013;102:86-95.
37. Rodbard D. Glycemic variability: measurement and utility in clinical medicine and research-one viewpoint. Diabetes Technol Ther. 2011;11:1-4.

38. Cavalot F. Do data in the literature indicate that glycaemic variability is a clinical problem? Glycaemic variability and vascular complications of diabetes. Diabetes Obes Metab. 2013;15:3-8.

39. Carlson AL, Mullen DM, Bergenstal RM. Clinical use of continuous glucose monitoring in adults with type 2 diabetes. Diabetes Technol Ther. 2017;19:S4-11.

40. Dandona P. Minimizing glycemic fluctuations in patients with type 2 diabetes: approaches and importance. Diabetes Technol Ther. 2017;19:498-506.

41. Lipska KJ, Krumholz HM. Is hemoglobin A1c the right outcome for studies of diabetes? JAMA. 2017;317:1017-8.

42. Ismail-Beigi F, Moghissi E, Kosiborod M, et al. Shifting paradigms in the medical management of type 2 diabetes: reflections on recent cardiovascular outcome trials. J Gen Intern Med. 2017;32:1044-51.

43. Lipska KJ, Yao X, Herrin J, et al. Trends in drug utilization, glycaemic control and rates of severe hypoglycemia 2006-2013. Diabetes Care. 2017;40(468-475):44.

44. Giorgino F, Bonadonna RC, Gentile S, et al. Treatment intensification in patients with inadequate glycemic control on basal insulin: rationale and clinical evidence for the use of short-acting and other glucagon-like peptide-1 receptor agonists. Diabetes Metab Res Rev. 2016;32:497-511.

45. Chrvala CA, Sherr D, Lipman RD. Diabetes selfmanagement education for adults with type 2 diabetes mellitus: a systematic review of the effect on glycemic control. Patient Educ Couns. 2016;99:926-43.

46. Rodbard D. Continuous glucose monitoring: a review of recent studies demonstrating improved glycemic outcomes. Diabetes Technol Ther. 2017;19:25-37. 